\title{
A SOLID-STATE MODULATOR FOR HIGH SPEED KICKERS*
}

\author{
J.A. Watson, E.G. Cook, Y.J. Chen, R. M. Anaya, B.S. Lee, J.S. Sullivan, S.A. Hawkins, F.V. \\ Allen, B.C. Hickman \\ Lawrence Livermore National Laboratory
}

C.A. Brooksby - Bechtel Nevada

\begin{abstract}
An all solid-state modulator with multi-pulse burst capability, very fast rise and fall times, pulse width agility, and amplitude modulation capability for use with high-speed beam kickers has been designed and tested at LLNL. The modulator uses multiple solid-state modules stacked in an inductive-adder configuration. It provides a nominal $18 \mathrm{kV}$ pulse with $+/-10 \%$ amplitude modulation on the order of several $\mathrm{MHz}$, rise times on the order of $10 \mathrm{nS}$, and can be configured for either positive or negative polarity. The presentation will include measured performance data.
\end{abstract}

\section{INTRODUCTION}

High-speed beam kickers are used to provide precision beam manipulation for high current electron beams. This technology has been demonstrated on the ETA-II accelerator at LLNL, and will be used on the $2^{\text {nd }}$ axis of the DARHT accelerator at LANL. A detailed treatment of the kicker used on the ETA-II accelerator can be found in [3]. The modulators used to drive the kicker employ inductive adder topology and MOSFET switches to meet the high current and fast risetime specification. They include amplitude modulation capability to compensate for transverse e-beam motion (from a variety of sources), as well as the dynamic response and beam-induced steering effects associated with the kicker structure.

\section{THE MODULATOR}

The modulator is a linear inductive adder, essentially a stack of transformers with the secondaries connected in series. The primary and secondary winding of each stage consists of a single turn to keep the leakage inductance small. High speed MOSFETS are used to switch energy stored in a capacitor into the primary of each transformer. The conduction interval of the MOSFETS is controlled by the width of the pulse on the gate (input) of the device, allowing for variable pulse width and burst capability ${ }^{[1]}$. In the adder configuration shown in Figure. 1, the output voltage on the secondary winding is the sum of all the voltages appearing on the primary windings. Each stage of the adder runs at a nominal voltage of $650 \mathrm{~V}$, resulting in 28 stages for the adder.

The modulators are capable of providing $10 \mathrm{nS}$ rise, 16

*This work was performed under the auspices of the U.S. Department of Energy by the University of California Lawrence Livermore National Laboratory under Contract No. W-7405-Eng-48
- 200nS wide pulses in a multi-pulse burst with a maximum total pulse width of 500nS per burst and an inter-pulse spacing of $400 \mathrm{nS}$ minimum. Figure 2 shows a typical single-pulse waveform for the solid-state modulator.
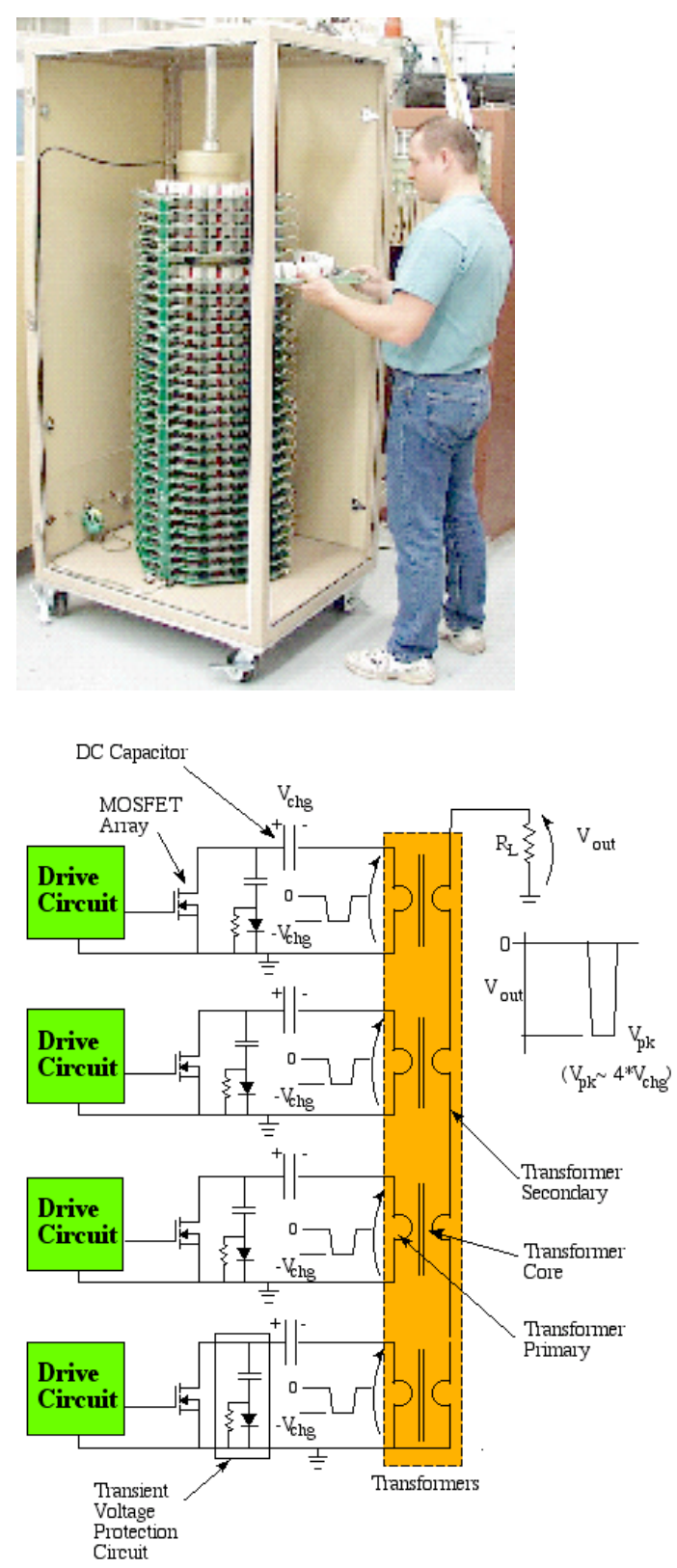

Figure 1 Solid-state modulator and block diagram 


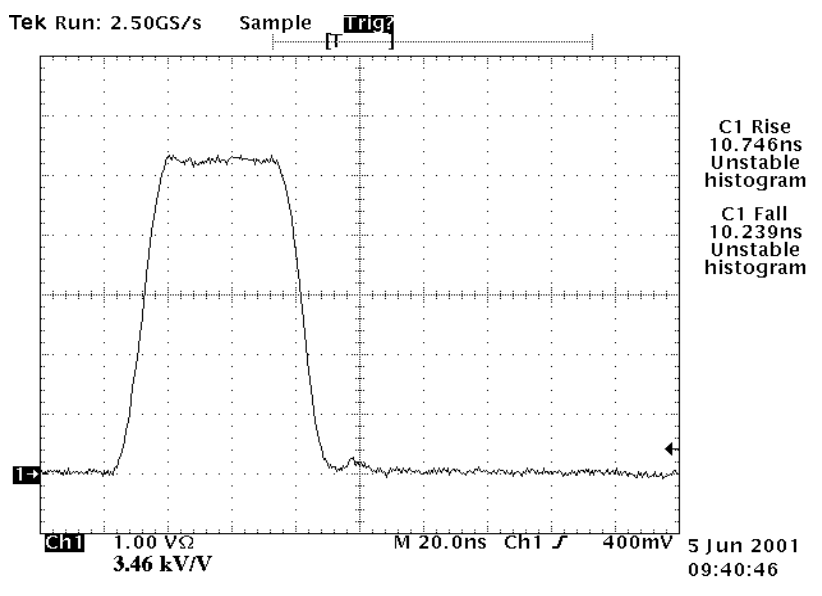

Figure 2 Modulator output voltage

\section{AMPLITUDE MODULATION}

To compensate for transverse e-beam motion, the amplitude of the pulse(s) applied to the kicker can be adjusted.

Amplitude modulation is accomplished by controlling individual stages in the adder structure. Seven stages, each with individual delay and pulsewidth control, are used to control the amplitude of the pulser. Two of the boards are charged to one fourth and one half, respectively, of the nominal charge voltage to achieve fine voltage control. The system is essentially a high voltage, ultra-high speed $\mathrm{D} / \mathrm{A}$ converter. While the turn-on time of the adder assembly is extremely fast (approaching $20 \mathrm{kV}$ in $10 \mathrm{nS}$ ), articulation of the amplitude is much more difficult as each modulation stage must switch the full output current of nearly 400A. We have achieved slew rates of over $50,000 \mathrm{~V} / \mu \mathrm{Sec}$ (linear ramp) using this approach.

\section{CONTROL SYSTEM}

The control system used to determine the desired pulser output voltage utilizes a feedforward design whereby the calculated voltage applied to the kicker plates is applied on the next iteration based on information from the current and past iterations. The beam is not actively controlled as it passes through the kicker; rather the voltage applied to the kicker is calculated based on the measured position of the beam on the previous pulse. There are two components to the control system, one that calculates the desired voltage to be applied to the kicker and the second to accurately control the output of the pulsers. The control bandwidth, expressed as the highest rate of transverse beam motion that can be corrected, is limited primarily by the bandwidth of the modulation stages in the pulsers. This bandwidth is on the order of $10 \mathrm{MHz}$.

\subsection{Beam Control Algorithm}

The beam control algorithm, or BCA, calculates the desired voltage to be applied to the kicker. The complete treatment of dipole steering through the kicker has no analytic closed form solution. A detailed treatment can be found in [2]. If we assume negligible beam-induced steering effects, we have a solution to the differentialintegral equation of the kicker as a function of time

$$
x(z=l, t)=\frac{c^{2}}{4 V_{0} l} \int_{t-2 l / c}^{t} V_{p}\left(t^{\prime}\right)\left(t-t^{\prime}\right) d t^{\prime}
$$

where $V_{p}(t)$ is the applied voltage and $c$ is the speed of light in free space.

The control system attempts to compensate for deterministic errors in beam position by applying a solution for $x(\mathrm{t})$ to the following beam pulse. The control system is iterative and adaptive, and can correct for effects that are deterministic but not correctly estimated by (1). As shown in Figure 3, an outer loop takes beam position data and calculates a desired pulser voltage waveform. An inner loop then attempts to match actual output of the pulser to the desired waveform.

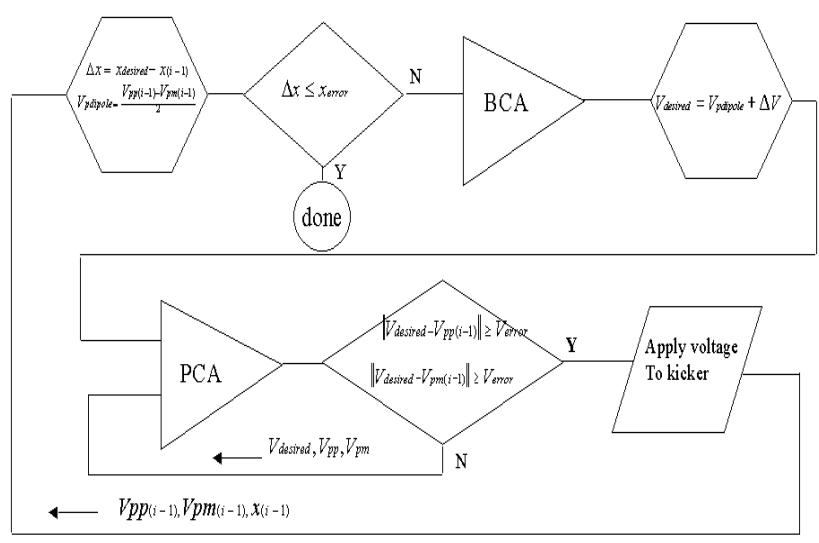

Figure 3 Kicker Control System

The control system has been tested on the ETA-II accelerator at LLNL. Figure $4 \mathrm{a}$ shows measured displacement of a kicked and unkicked beam. Figure $4 \mathrm{~b}$ shows the amplitude of the pulse applied to the kicker to achieve this beam displacement. These tests were performed with hard tube modulators driving the kicker. The solid-state modulators have yet to be tested on ETAII.

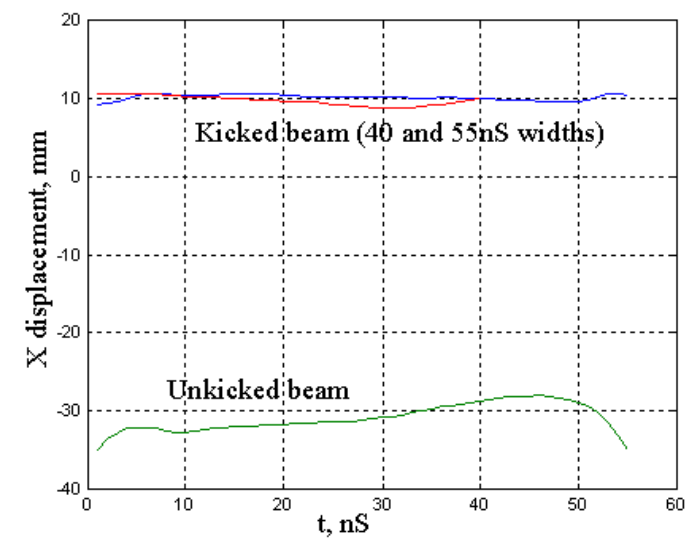

Figure 4a kicked and unkicked beam position for 40 and $55 \mathrm{nS}$ wide beam pulses on ETAII 


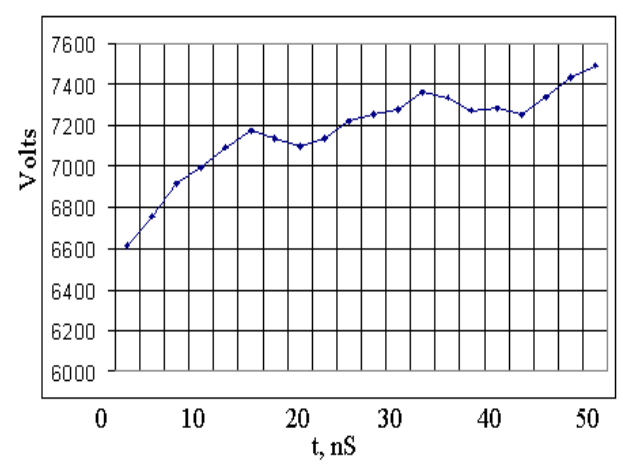

Figure $4 \mathrm{~b}$ Modulator output

\subsection{Pulser Control Algorithm}

The pulser control algorithm, or PCA, uses simple proportional-integral feedback. The output of the PCA feeds a high-speed digital pattern generator (DPG) to control the seven modulation stages in the adder. The conduction interval of the MOSFET switches is not linearly related to the input, or desired pulsewidth for narrow pulses due to characteristics inherent with the devices. Figure 5 shows the relationship between output pulsewidth to input (gate) pulsewidth for the seven modulation stages. Lookup tables were evaluated to compensate for this effect but quickly abandoned due to their cumbersome and inflexible nature. Instead, we have implemented a more general approach that adds a minimum duration to the input pulsewidth. Additionally, to help compensate for the inherent propagation delay in the switches, we included a derivative feedforward term as shown in figure 6 . This term adds a derivative component to the input of a software digitizer (the "A/D" in figure 6) and also adds directly to the pulsewidth of the control lines to the two bottom levels of the modulation stages.

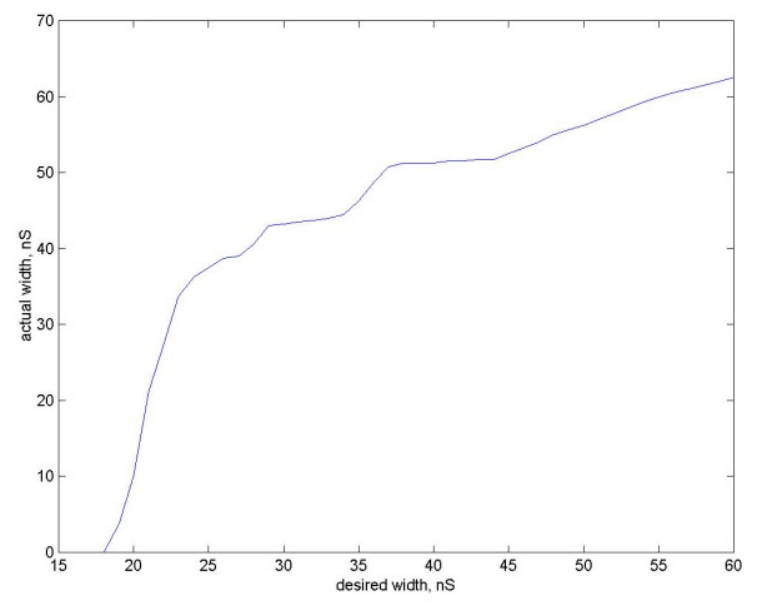

Figure 5 MOSFET switch output pulsewidth versus desired width

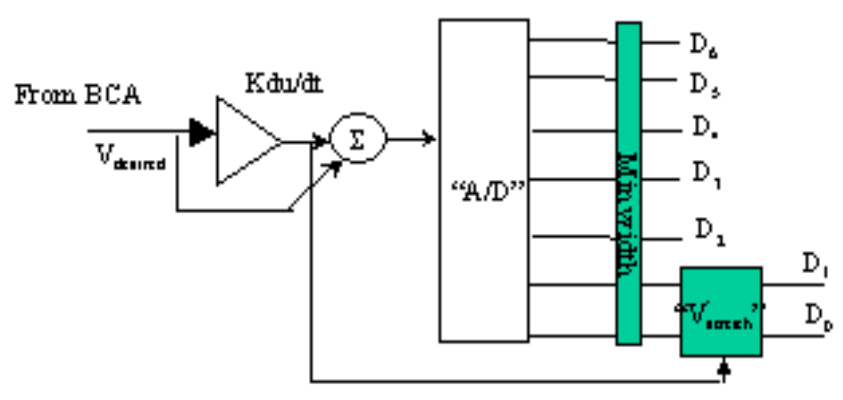

Figure 6 Subsection of the PCA

\section{PERFORMANCE}

We have done extensive testing of the solid-state modulator into a $50 \mathrm{ohm}$ load. We will be conducting full-scale integrated tests this summer on ETA-II of several components designed and built at LLNL for the DARHT $2^{\text {nd }}$ axis accelerator, including the kicker, septum and associated downstream transport components, the solid-state modulators, and the control system. Figure 7 shows a typical multi-pulse burst output for the solid-state modulator. The green trace in figure 7 shows the desired pulse parameters (shifted in time for clarity). The first pulse in the burst is approximately $20 \mathrm{nS}$ wide at $90 \%$ peak. The pulses shown here are spaced at a uniform $600 \mathrm{nS}$, however inter-pulse spacing is arbitrary subject to a $400 \mathrm{nS}$ minimum.

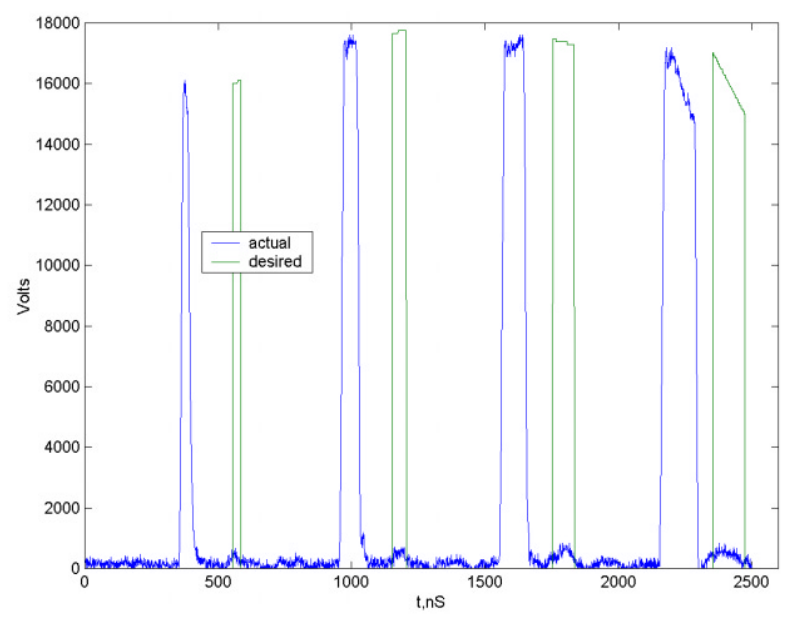

Figure 7 Four-pulse burst with amplitude modulation

\section{REFERENCES}

[1] Ed Cook, et al. "Inductive-adder Kicker Modulator for DARHT 2" Linac2000, Monterey, CA

[2] Y.J. Chen "Notes on the Kicker Control System" USPAS 2000W, Tucson, AZ

[3] Y.J. Chen et al. "Precision fast kickers for kiloampere electron beams" 1999 Particle Accelerator Conference, New York, NY 